\title{
Models That Matter: White Matter Stroke Models
}

\author{
Elif G. Sozmen • Jason D. Hinman • \\ S. Thomas Carmichael
}

Published online: 24 February 2012

(C) The American Society for Experimental NeuroTherapeutics, Inc. 2012

\begin{abstract}
Summary Stroke is a devastating neurological disease with limited functional recovery. Stroke affects all cellular elements of the brain and impacts areas traditionally classified as both gray matter and white matter. In fact, stroke in subcortical white matter regions of the brain accounts for approximately $30 \%$ of all stroke subtypes, and white matter injury is a component of most classes of stroke damage. However, most basic scientific information in stroke cell death and neural repair relates principally to neuronal cell death and repair. Despite an emerging biological understanding of white matter development, adult function, and reorganization in inflammatory diseases, such as multiple sclerosis, little is known of the specific molecular and cellular events in white matter ischemia. This limitation stems in part from the difficulty in generating animal models of white matter stroke. This review will discuss recent progress in studies of animal models of white matter stroke, and the emerging principles of cell death and repair in oligodendrocytes, axons, and astrocytes in white matter ischemic injury.
\end{abstract}

Key Words Ischemia - oligodendrocyte - oligodendrocyte progenitor cell $\cdot$ mouse $\cdot$ rat $\cdot$ axoglial unit $\cdot$ endothelin

\section{Clinical Aspects of White Matter Stroke}

Subcortical white matter stroke constitutes 15 to $25 \%$ of all stroke subtypes $[1,2]$. These lesions encompass small

E. G. Sozmen · J. D. Hinman • S. T. Carmichael $(\bowtie)$

Department of Neurology,

David Geffen School of Medicine at UCLA,

Los Angeles, CA 90095, USA

e-mail: scarmichael@mednet.ucla.edu infarcts in deep penetrating vessels in the brain [3-5]. In traditional stroke subtyping, subcortical or white matter stroke includes infarcts in the white matter deep to the cortex in humans, and also small basal ganglia, thalamus, and brainstem strokes or "lacunar" infarctions. Recent studies indicate distinct clinicopathological entities between subcortical white matter versus brainstem, thalamic, or basal ganglia lacunar infarctions. Subcortical white matter infarctions are more closely related to microvascular stroke risk factors, such as hypertension and diabetes, and appear to involve a spectrum, especially as seen in magnetic resonance imaging (MRI), of ischemic white matter hyperintensities to small necrotic cavities [4]. Pathologically, white matter strokes progress and enlarge: repeat MRI imaging shows that new white matter strokes develop within pre-existing lesions and also are associated with adjacent lesions in $71 \%$ of cases [4]. Interestingly, this tissue progression from recent MRI studies resembles the original description of the pathology of lacunar infarcts from Dechambre in 1838 of a central lesion of "softening," which is then absorbed to produce the necrotic cavity [6]. This current and historical perspective indicates that the specific ischemic focus in white matter is distinct from that of large vessel disease, and is a progressive focal lesion.

Subcortical white matter stroke has devastating clinical consequences. This was first cohesively presented by Pierre Marie, who described the accumulation of small clinical strokes into a progressive state, an état lacunaire, of motor slowing and global intellectual deterioration [7, 8]. Small white matter or lacunar infarcts produce hemiparesis or hemisensory loss with incomplete recovery, and they accumulate to produce gait abnormalities, verbal processing deficits, and difficulties in executive functioning that present as vascular dementia $[9,10]$. In prospective studies, small white matter strokes are associated with progressive cognitive decline [11]. 
The incidence of subcortical white matter stroke increases with age and is expected to increase in prevalence with the aging population [12]. With untreated small vessel stroke risk factors, such as diabetes and high blood pressure, white matter lesions accumulate and secondary brain atrophy progresses [13]. This clinical, pathological, and imaging data identifies 4 alarming aspects of white matter stroke: it has an increasing incidence, progresses in the face of modest risk factors, it generates a vulnerable peri-infarct region that can develop further infarction, and it disrupts neuronal connections to cause substantial disability. The development of appropriate experimental models of white matter stroke is essential to better understand its progressive nature and to develop novel therapeutics that can ameliorate its devastating clinical consequences.

\section{Goals of Modeling White Matter Stroke}

Clinically, most white matter infarcts are believed to be secondary to vascular occlusion from changes in small vessels, as well as endothelial cell dysfunction [14, 15], although the underlying mechanisms for such vasculopathies may vary. As such, replicating the white matter ischemic damage seen in humans has proven to be relatively troublesome in experimental animals. Studies that partially model central nervous system (CNS) white matter ischemia in vitro (such as optic nerve anoxia/reoxygenization) have been useful in identifying ion channel abnormalities during oxygen deprivation $[16,17]$. However, these studies fail to replicate the complexity of the human disease by design, given the absence of an inflammatory response, blood flow, and lack of a neurovascular unit. Small animal models that feature some of the parenchymal and/or microvessel pathology associated with human white matter stroke remain crucial to understanding the disease process and developing new interventions.

Distinct histological changes are found within ischemic white matter rarefaction as: focal edema, demyelination, axonal damage, loss of oligodendrocytes, and local activation of astrocytes and microglia $[18,19]$. Unlike gray matter, the window of therapeutic opportunity for axonal rescue and early changes in the penumbra have never been defined for white matter after the onset of ischemia. The time course of axonal injury and myelin disruption is vital to estimate an appropriate course of action. In the case of the lacunar infarct, small vessel pathology is highlighted as a major histological finding [14] in that the initiating step appears to be disruption of the blood- brain barrier (BBB), even though the late event causing the lesion is considered ischemic $[14,20]$. These different pathological mechanisms leading to white matter stroke in humans, from more chronic alterations in the BBB to acute vascular occlusion, indicate that multiple models may be necessary to replicate the complete spectrum of white matter stroke lesions.

With that in mind, a useful representation of white matter stroke can roughly fall into 2 categories (Table 1). The first consists of focal white matter stroke, which models white matter lesions secondary to acute ischemia and/or occlusion of deep penetrating arterioles. The second set of models is consistent with global, chronic conditions, such as dysregulated cerebral blood flow (CBF), which results in generalized white matter disease. Modeling both states can provide a comprehensive picture of the initiating factors involved, and of the cellular interactions that play roles in white matter stroke progression. In addition to similar pathology, appropriate behavioral tests conducted on small animals are invaluable to assess functional impairment and to ascertain whether the lesion created in the model is relevant to human cases of white matter stroke. Finally, rodent models of white matter stroke, particularly mice, are preferred due to the availability of sequence data, antibodies, and genetically modified strains.

\section{Focal White Matter Stroke Models}

\section{ET-1/Vasoconstriction Method}

Although it is not possible to duplicate all components of human white matter stroke in an animal model, it is essential to control infarct location. Stereotaxic injection of vasoconstrictive agents allows such spatial control to produce confined white matter lesions. One of the most commonly used vasoconstrictive substances is endothelin-1 (ET-1). Endothelin is a naturally produced vascular endothelial cell peptide that may have paradoxical vasoconstrictive or vasodilatory activity when binding the respective endothelin receptors, ETA-R and ETB-R [21]. ET-1 is used to significantly reduce local blood flow to levels that produce ischemic injury when injected directly into parenchy$\mathrm{ma}$ [22], and to induce precise and reproducible focal ischemic lesions in gray or white matter without disruption of the BBB [23]. This method has been largely used in modeling disease in rats.

When injected into rat subcortical white matter and striatum, ET-1 causes a short-lived $(3 \mathrm{~h})$ decline in regional $\mathrm{CBF}$ as measured by MRI and signal attenuation of an intravenous contrast agent [23]. Approximately a $60 \%$ reduction in CBF leads to a hyperintense area detected by T2-weighted MRI within $24 \mathrm{~h}$ of injection [23]. The regional blood flow is restored by $3 \mathrm{~h}$. Interestingly, ischemia induced by ET-1 administration does not appear to affect BBB integrity. Contrast-enhanced T1-weighted images show that 


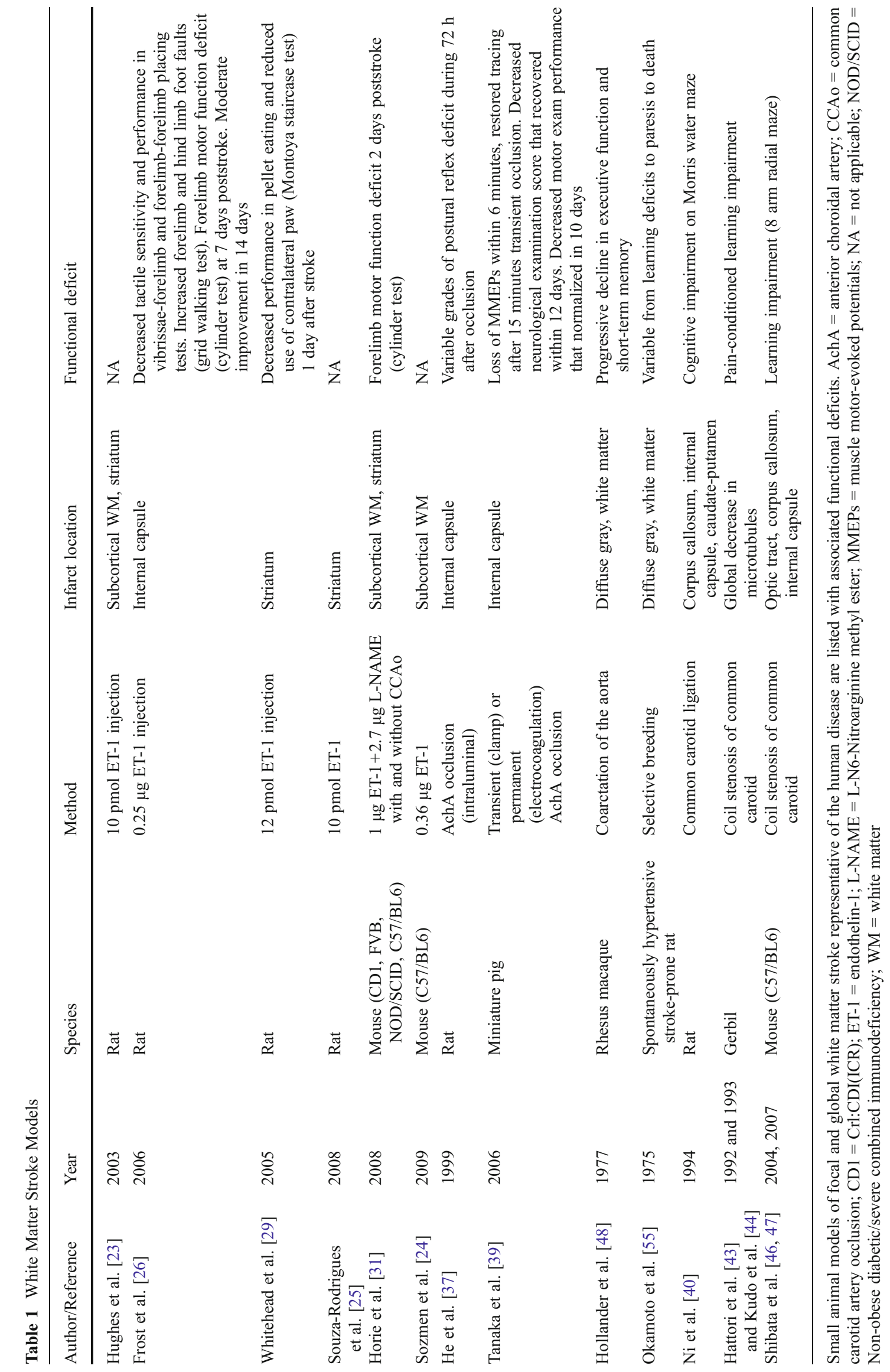


extravasation of the contrast agent did not occur within the injection and administration of a BBB marker confirmed that there is no loss of this barrier after lesion formation [23]. ET-1 mediated ischemia likely leads to cytotoxic edema that is reflected as white matter hyperintensities in T2-weighted MR scans shortly after the injection $(24 \mathrm{~h})$. Similar white matter hyperintesity is observed in a subcortical mouse model of ET-1, evident at 2 days postinjury [24].

Although the ET-1 model would not be suitable to model vasogenic edema, the histological studies show significant similarities to human white matter stroke. ET-1 leads to mixed gray and white matter lesions in striatum with decreased detectable myelin basic protein, in addition to prominent neuronal loss $[23,25]$. In turn when injected directly into the subcortical white matter, ET-1 leads to myelin disruption without overt loss of myelin [23]. On the other hand, stroke induced by ET-1 injection into the white matter of the internal capsule indicates a well-demarcated, demyelinated region and necrosis after a 14-day survival period [26]. In the mouse model, a small area of decreased myelin is detected at 1 day that later develops into a larger region of diminished myelin basic protein staining from 14 to 28 days survival times [24].

Axonal injury is another hallmark of white matter stroke that is replicated by ET-1 induced ischemia. Amyloid precursor protein (APP) accumulation in axonal end bulbs has been used as a reflection of axonal damage in other models of brain injury [27]. Direct application of ET-1 into cortical white matter is shown to cause significant axonal disruption, detected as APP reactivity highest at $24 \mathrm{~h}$ and persisting at lower levels at 7 days [23]. In this model, the authors conducted measurements of the APP-positive area surrounding the injection site and described a shrinking zone of interrupted axonal transport with this marker during the course of 7 days. ET-1 injection into subcortical white matter in the mouse results in a region void of neurofilaments and axons [24]. However, there is a peri-infarct region of dynamic tissue change adjacent to the stroke core. In mouse white matter stroke, the region of demyelination is larger than the region of axonal neurofilament loss, suggesting a candidate region for remyelination and repair [24]. Interestingly, axonal injury is evident prior to any clear changes in myelin in a rat model of white matter ischemia [23]. In agreement with this observation, Sozmen et al. [24] reported signs of early axonal damage that were evident by ultrastructural electron microscopy (EM) studies at $24 \mathrm{~h}$, whereas myelin sheaths appear intact within the infarct. At later time points, concomitant axonal and myelin damage are observed [24]. Histological and ultrastructural analysis of white matter stroke suggest that the peri-infarct zone evolves, thus allowing salvage of this tissue if appropriate treatments are identified.

Controversial results have been reported regarding the inflammatory response following ET-1 induced focal ischemia. Hughes et al. [23] reported a prominent macrophage/microglia response, but an absence of neutrophil recruitment in striatum and subcortical white matter at 3 days post-ET-1 injection in rat. On the contrary, Souza-Rodrigues et al. [25] showed a prolific inflammatory response of both neutrophil and mononuclear leukocytes that is more acute ( $24 \mathrm{~h})$ using the same amount of ET-1 [25]. The ET-1 subcortical stroke in the mouse also causes early microglia/macrophage infiltration, although neutrophil involvement has not been investigated [24]. The inflammatory cells in this model were localized to axons with SMI-32 positive neurofilaments soon after ET-1 injection. SMI-32 is a marker for dephosphorylated neurofilament protein that is sparsely present in the normal brain, but marks areas of axonal damage after injury [28]. The dynamics between the type of inflammatory cells and severity of damage warrants further investigation.

Astrocyte activation appears to be a delayed event relative to axonal damage in ET-1-induced subcortical/white matter stroke models. Glial fibrillary acidic protein (GFAP)-positive cell numbers and process hypertrophy are prominent later than $24 \mathrm{~h} \mathrm{[23].} \mathrm{The} \mathrm{glial} \mathrm{response} \mathrm{may} \mathrm{very} \mathrm{well} \mathrm{be} \mathrm{injection} \mathrm{site}$ dependent. For instance, in ET-1 stroke in the striatum GFAPpositive cells are in the surrounding tissue [23]. In contrast, injection into cerebral white matter did not change the cell numbers but caused an overall hypertrophy of GFAP-positive processes. Conversely, a prominent increase in both the density and process thickness are observed in the mouse model of white matter ET-1 injection [24]. In addition, striatal and cerebral white matter infarcts created by ET-1 indicate a persistent GFAP-positive cell response up to 21-day and 28day survival time, respectively [24, 29].

Overall, there are fewer reports using ET-1 to induce cerebral ischemia in mice, in part, because the ability of ET-1 to induce vasoconstriction is significantly lower in mice [30]. In the study carried out by Horie et al. [31] intrastriatal microinjection of $1.0 \mu \mathrm{g}$ ET-1 alone failed to cause a distinct lesion in multiple strains of mice. Furthermore, the injury is proven to be independent of ET-1 dose, because increasing the injection dose only contributed to postoperative mortality and not lesion formation. It is unclear why there is such a large discrepancy in the ability of ET-1 to cause infarcts in rats versus mice, since the ET-1 peptide sequence is highly conserved between the 2 species. Differences in endothelin receptor isoform expression may account for poor ET-1-mediated vasoconstriction. For instance, Wiley et al. [32] reported that the mouse brain expresses twice as many of ETB-R than ETA-R. As such, ET-1 administration could have a predominantly vasodilatory effect in mice. For that reason, Horie et al. [31] combined injection of nitric oxide synthase (NOS) inhibitor L-N6-Nitroarginine methyl ester (L-NAME) (to counter ET-1mediated vasodilation) and/or common carotid occlusion to produce striatal infarcts. Yet, the lesion size was still minimal and the effect was found to be mouse strain-dependent. In contrast, a white matter stroke model generated by ET-1 injection into subcortical white matter in C57/BL6 mice [24] 
shows lesions that mimic small lacunar infarcts. Similar to Horie et al., [31] a dose-dependent mortality was seen, however, a significantly lower amount (360 ng) was sufficient to cause the white matter infarct. This additional discrepancy of tissue dependent ET-1 effects further complicates the modeling of white matter stroke in mice.

Finally, a major drawback of using endothelins to model white matter stroke is the receptor activity in various cell types in addition to endothelial cells. ET-1 has been shown to exert a direct effect on the reactive nature of astrocytes via ETB receptors after acute brain injury [33, 34]. Previous studies have noted that ET-1 has direct effects on neurons and astrocytes affecting excitability, gap junctions, and gliosis [35]. Furthermore, oligodendrocyte progenitors are shown to respond to endothelins and alter their fate decisions when ligand binding occurs [36]. The direct action of ET-1 with cells that would be expected to have a role in neural repair and recovery in stroke makes the use of ET-1 as a stroke agent problematic.

\section{Anterior Choroidal Artery Occlusion Method}

The anterior choroidal artery (AchA) is a branch of the internal carotid artery that arises just proximal to the carotid bifurcation into middle and anterior cerebral arteries [37]. Intraluminal occlusion of the AchA in the rat creates an ischemic core that involves the internal capsule, although the infarct size may vary and the hypothalamus may also be involved [37, 38]. 2,3,5-triphenyltetrazolium chloride (TTC) staining and cell death assays show a successful modeling of capsular lacunar infarct with this method.

Tanaka et al. [39] developed a relevant gyrencephalic brain model of internal capsule lacunar infarcts by AchA occlusion in the miniature pig. Diffusion and T2-weighted MRI demarcate ischemic damage at $24 \mathrm{~h}$ after permanent occlusion. Histological studies evaluated the extent of white matter injury dynamic changes in the lesion. Luxol blue labeling of white matter revealed damaged myelin sheaths with vacuoles. Through histology, the authors defined areas of the ischemic lesion as the ischemic core, an irreversibly damaged area, a peri-infarct area, and a marginal zone. Axonal damage measured by APP-positive bulbs and axonal swellings detected spreading damage into peri-infarct areas during the first week after ischemia. The core contained necrotic astrocytes and oligodendrocytes after 1 week of ischemia. The peri-infarct area consisted of GFAP-positive astrocytes from early stages, which enlarged by 4 weeks. A notable finding of this study is that small vessels were found to be highly resistant to ischemic stress. The number of small vessels in the capsular infarct was not different from control animals even in the chronic phase.

Notably, ultrastructural analysis of the infarct in the mini pig model highlights the differences in the predetermined lesion zones, which was not possible to observe in postmortem human infarcts. Twenty-four hours after ischemia, the ischemic core contains both axons and myelin that were morphologically destroyed. One week later, only myelin sheath debris was visible that was phagocytosed by infiltrated macrophages. In the irreversibly damaged area at $24 \mathrm{~h}$ after ischemia, signs of edema, disrupted axonal alignment, and aberrant myelin sheaths were observed. Swollen axons with myelin sheaths were present in the peri-infarct. In the marginal area, there were no initial significant abnormal findings, except a low grade of swollen axons and exudate. One week later, swollen axons included many organelles with fragile myelin sheaths. Overall these findings indicate progressive expansion of ischemic damage to the marginal area. Furthermore, the ultrastructural analysis suggests that axonal damage results in the instability of the myelin sheath and subsequent demyelination. These findings are partially in agreement with the early and late changes found in the mouse subcortical stroke model, even though the infarct zones were not established EM [24].

\section{Global Brain Ischemia as White Matter Stroke}

Several approaches have modeled white matter stroke by lowering global brain perfusion either via direct proximal vessel manipulation or induced systemic hypertension. Each use different animal models and have variable effects on white matter.

\section{Carotid Occlusion/Stenosis Models}

Permanent bilateral common carotid occlusion by ligature in rats resulted in $\sim 50 \%$ reduction in cortical blood flow [40, 41]. This global cerebral hypoperfusion produces an apparent loss of cellularity, vacuolation of myelin, and astroglial reactivity within major white matter structures, such as the optic nerve, corpus callosum, and internal capsule as early as 7 days after occlusion [41, 42]. This also produces measurable cognitive deficits (for more detail see Hainsworth and Markus [5]). Although this model does produce white matter lesions and cognitive deficits, the high postoperative mortality rate (10$20 \%$ ) and the rapidity of lesion development do not mimic the human condition in which white matter ischemic lesions progress slowly and are rarely diffuse, but rather focal with regional predominance.

Bilateral common carotid stenosis has been reported in both gerbil (with an incomplete circle of Willis) [43-45] and mouse [46, 47]. The stenosis is produced by placing a surgical coil around the common carotid arteries bilaterally, which incompletely obstructs the vessel and produces $\sim 25 \%$ reduction in cerebral blood flow in both species [44, 46]. In the gerbil, this produces necrotic foci in both gray and white 
matter structures with dendritic swelling and myelin loss [43], predominantly in a pattern of watershed ischemia [44]. The lesions develop more slowly at $>8$ weeks and are associated with learning impairment [44]. This contrasts with the acute development of white matter stroke in the human, although long-term progression of white matter ischemia has been described [4].

In the mouse, bilateral common carotid occlusion with coils produces similar ischemic lesions to those present in the gerbil; within the optic tract, corpus callosum and internal capsule with myelin loss begin at 14 days [46]. In these C57/ BL6 mice with $\sim 25 \%$ reduction in cerebral blood flow, Shibata et al. [46, 47] report no objective cortical infarcts, hemorrhages, or significant change in histological stains within the cortex at 30 days. This model is useful in that it uses C57/BL6 mice, allowing for the application of mouse transgenic paradigms to be easily studied in the context of cerebral hypoperfusion. Whether the cerebral cortex shows other phenotypic hallmarks of ischemic injury (such as neuronal nitric oxide synthase (nNOS), c-fos, c-jun induction, or hydroxyl radical formation) rather than histopathological changes, remains to be established. Notably, there is a similar 10 to $20 \% 30$-day postoperative mortality rate following bilateral carotid stenosis, raising concerns that this model entails a degree of direct, generalized brain damage that is not present in human white matter stroke.

\section{Systemic Hypertension Models}

Long-standing systemic hypertension is a known risk factor in humans for the development of white matter ischemic lesions on MRI. Several models of systemically hypertensive rodents and primates demonstrate variable degrees of CNS pathology.

Surgical narrowing of the proximal aorta by 70 to $80 \%$ in rhesus macaques produces a systemic hypertension of $\sim 180$ / $110 \mathrm{mmHg}$ associated with retinopathy and hypertrophic cardiomyopathy, similar to that seen in humans with longstanding hypertension [48]. These primates develop ageassociated focal cerebrovascular ischemic lesions with resultant loss of neurons and myelin associated with a local inflammatory response marked by astroglial proliferation and microglial influx [49, 50]. The lesions are diffuse and reported in hippocampus, cerebral cortex, striatum, brainstem, and cerebellum, and thus they are not restricted to white matter structures.

Rats transgenic for an inducible variant of the mouse renin gene (under the control of the cytochrome $\mathrm{P} 450$ promoter) develop systemic hypertension and related organ effects within roughly 2 weeks of transgene induction [51, 52]. Interestingly, no brain pathology was noted at 14 days. Transgenic induction of hypertension, plus the addition of normal saline to drinking water, produced focal brain hemorrhagic lesions.
This was associated with increasing mortality [52]. Mice transgenic for human renin and human angiotensinogen require a "second hit" for the development of significant systemic hypertension and CNS pathology $[53,54]$. Following treatment with a high-salt diet and L-NAME, these $\mathrm{R}+/ \mathrm{A}+$ mice develop progressive systemic hypertension and multiple foci of small hemorrhagic lesions with brainstem predominance [54]. This model requires both genetic modulation and systemic pharmacologic vasoconstriction and results in lesions that are atypical for the human condition of white matter stroke.

Spontaneously hypertensive Kyoto rats with stroke phenotype were selectively bred and developed progressive arterial hypertension beginning at 4 weeks of age and reaching a plateau at 20 weeks $[55,56]$. Systolic pressures peaked at $\sim 220 \mathrm{mmHg}$ and the rats developed a progressive arteriopathy, which shares several pathologic features with human small vessel disease. These features include wall thickening of small penetrators, replacement of smooth muscle by fibrous material, and local erosion of the BBB $[56,57]$. The CNS pathology observed is age-dependent, first appearing at 12 weeks and reaching its maximum at 30 weeks $[56,58]$. Ischemic lesions occur in the striatum, as well as frontal, parietal, and occipital cortices. White matter lesions are present with rarefaction of myelin and local edema, a percentage of which demonstrate associated hemorrhage [59-61]. These rats have been extensively studied with agents to prevent or ameliorate the stroke phenotype (for more detail see Hainsworth and Markus [5]).

One main limitation of these models of systemic hypertension is the animal-to-animal variability in CNS pathology (i.e., lesion size, presence of hemorrhage, neuroanatomical location). This variability makes it difficult for systematic study of a variety of features that will undoubtedly prove important to the pathophysiology of white matter stroke. These features include local oligodendrocyte response to ischemia, the response of the axoglial unit to ischemia, axonal regrowth, and oligodendrocyte precursor repopulation.

\section{Arteriopathy Models}

Clinical interest in inherited white matter lesions seen in cerebral autosomal dominant arteriopathy with subcortical infarcts and leukoencephalopathy raise interest in modeling this disease in rodents. Mice transgenic for the Notch-3 mutations associated with cerebral autosomal dominant arteriopathy with subcortical infarcts and leukoencephalopathy in humans develop progressive arteriopathy, but show no clear evidence of CNS pathology [62]. Similar clinical interest in a rare genetic form of white matter stroke with point mutations in type IV collagen [63] drove the development of heterozygote mice with a large deletion in type IV procollagen $\alpha 1$. These mice develop cerebral hemorrhages 
as pups and $50 \%$ of them die by postnatal day 1 . Based on these reports, neither of these models appears suitable for the systematic study of adult onset ischemic white matter disease in humans.

\section{Functional Deficits Associated with White Matter Stroke}

Behavioral outcomes of white matter infarcts have provided insights in the time course of recovery and persistent injury. Frost et al. [26] presented experimental animal data examining the behavioral outcome after capsular ET-1 injection. The internal capsule is a common site for clinical strokes in the cerebral white matter in humans, and it was an initial focus for studies of functional network changes during recovery [64]. An infarct in this location results in degeneration of a significant number of descending fibers from the motor cortex and ascending fibers to the somatosensory cortex. After a 14-day survival period, the injury results in measurable sensorimotor deficits within 1 week, including decreased forelimb responses to vibrissae and forelimb stimulation, decreased forelimb tactile sensitivity, and abnormal limb control in gait. There is a later recovery of some of these deficits. AchA occlusion and capsular/hypothalamic infarcts in the rat [38] also produce behavioral deficits. The infarct size and location is more variable in this model than with the ET-1 method [38]. Accordingly, the postural reflex test showed varied grades but a consistent deficit in the postural reflex test that persisted for $72 \mathrm{~h} \mathrm{[37].}$

The AchA infarct in the mini pig produces measurable deficits in the acute and chronic phases of capsular ischemia [39]. Muscle motor-evoked potentials were used to evaluate corticospinal tract function during the acute phase of ischemia. Muscle motor-evoked potentials were diminished within 6 minutes but were successfully recovered after 15 minutes of transient occlusion. Total neurological and motor exams scores were recorded during the chronic phase of ischemia. Lacunar infarcts that were created to follow permanent occlusion were associated with motor deficits during the first 7 days of occlusion. Interestingly, the occlusion group recovered to normal scores by day 10 . The neurological deficit score showed no significant difference between ischemia and sham-operated groups at 12 days, even though the occlusion group showed a higher deficit within the first week. Such established spontaneous recovery is a main difference relative to human stroke, although more stringent behavioral tests may be needed to assess the extent of injury and recovery.

In contrast to the capsular infarcts, there is less known about the functional deficits associated with subcortical/ striatal infarcts. ET-1 injection into rat striatum/cerebral white matter results in reduced pellet eating behavior and decreased use of the contralateral paw in the Montoya staircase test during the $24 \mathrm{~h}$ after stroke, whereas no deficit is reported after this time point [29]. Compensation in behavior of the ipsilateral paw may be responsible for these results. Subcortical/striatal injury in the mouse also indicates decreased forelimb motor function after 2 days of ET-1 injection [31]. Further time point data is not available to assess long-term effects of these infarcts. Additional sensorimotor tests may be needed to profile the implications of striatal infarcts.

\section{Neural Repair in White Matter Stroke}

There is limited to no data available regarding remyelination and axonal regeneration following white matter stroke. Although, oligodendrocyte regeneration is reported in the mouse model of subcortical stroke [24], it is unclear if remyelination takes place in the relatively preserved peri-infarct area. In animal models of multiple sclerosis or primary (toxic) white matter injury, oligodendrocyte progenitor cells (OPCs) respond with cell division, migration to lesion sites, and differentiation into myelin-producing mature oligodendrocytes [65] as reviewed in Franklin and Ffrench-Constant [66]. This response has led to the characterization of injuryinduced OPCs as "reactive OPCs" [67]. Environmental signals govern the behavior of reactive OPCs following injury [68-70]. The induction of the reactive state of OPCs in demyelinating injury involves specific inflammatory cytokines, such as bone morphogenic protein (BMP)-4 and the heparin-binding growth factor pleiotrophin, insulin-like growth factor signaling, notch signaling, and the transcription factors (i.e., Nkx2.2, Olig1, and Olig2) [65, 67, 68, 70, 71]. Oligodendrocytes undergo profound morphological changes after stroke that suggest dysfunction and recovery [72]. However, it is not known if these same molecular systems play a role in white matter stroke, which differ from models of demyelinating injury in critical aspects of the previously mentioned environmental cues (i.e., the nature of the inflammation, the involvement of hypoxic and free radical events, and the lack of a toxic oligodendrocyte cell death that is present in many of the demyelinating models). In the same way that we have begun to understand the putative neural mechanisms involved in recovery from gray matter infarcts, we may be able to examine the reorganization and repair processes that take place after disruption of subcortical white matter, while the cortex remains intact.

\section{Cell Death in White Matter Stroke}

In white matter stroke, the neuroanatomical structures affected by ischemia are diverse and typically include: capillary endothelium, axonal projections, oligodendrocyte cell bodies and myelinating processes, and astrocytes 
interacting with both the endothelium (as the BBB) and axons (at nodes of Ranvier), as well as scattered microglia. Within this microenvironment, cell death can be measured in a variety of ways. Endothelial cells may undergo necrosis and result in focal hemorrhage. Oligodendrocyte cell bodies can die and thus lose their ability to maintain myelin loops. Isolated loops of myelin can lose their energy source and retract [72]. Focal segments of axons deprived of ATP undergo a distinct molecular process leading to Wallerian degeneration (for more detail see Stirling and Stys [73]). Astrocytes and microglia appear to be more resistant to ischemia and typically survive to play a significant role in the subsequent intrinsic inflammatory response to white matter stroke.

There is an extensive literature on the vulnerability of oligodendrocytes, both mature and immature to the effects of ischemia. This has been reviewed elsewhere [74, 75] and stems in large measure from in vitro models of white matter insult, which lack vascular and inflammatory contributions. In brief, the mature oligodendrocyte appears to be at least as susceptible to ischemia as a mature neuron. In a rat model of middle cerebral artery occlusion (MCAO), oligodendrocytes undergo cell swelling with $3 \mathrm{~h}$ of increased tauimmunoreactivity, and eventually demonstrate evidence of apoptosis [76, 77]. By $6 \mathrm{~h}$ after MCAO, myelin appears vacuolated and dissociated from the axolemma [77]. More recently, McIver et al. [72] used the rat model of MCAO to show that oligodendrocytes die and retract processes within $24 \mathrm{~h}$ of stroke. They also demonstrated that 1 week after MCAO, oligodendrocytes repopulate and extend processes in areas of ischemic white matter affected by stroke. However, middle cerebral artery occlusion damages both white matter and the neurons that give rise to the axons passing through the white matter, so it is not clear whether pathology seen in traditional stroke models is due to selective white matter injury. In the models reviewed here, only a few studies have examined oligodendrocyte responses to stroke. In focal ET-1-induced white matter stroke in the mouse, cells labeled with apoptosis inducing factor peak at 1 day after stroke within surrounding white matter. In situ, DNA nicking was also evident within oligodendrocytes after 1 day [24]. Focal injection of ET-1 into rat subcortical white matter produces a rapid decrease in tau-positive oligodendrocytes between 6 and $24 \mathrm{~h}$ and by 7 days, and these cells were basically gone [23]. In the various models of global brain ischemia, no objective measurements of oligodendrocyte cell death have been performed.

Arguably, axonal death and decay may be the most important aspect of white matter stroke. An intact axon without its myelin (initially) remains functional [77] and may be remyelinated under the right circumstances. For axonal loss to recover, it will require both axonal regrowth and remyelination within a glial scar, something that has proven to be challenging in multiple models of white matter injury. In the rat model of ET-1-induced capsular white matter stroke, no objective study of cell death has been reported, but after 14 days there is focal loss of axons and myelin in the region of injection, as measured by histology [26]. Focal injection of ET-1 into rat subcortical white matter produces axonal swellings, as measured by APPpositive inclusions [23]. In the mouse model of focal ET-1 injection, axonal blebbing was seen along with a loss of neurofilament staining within the stroke core after 7 days [24]. An important issue for the future in this field of cell death in white matter stroke is the fundamental difference between "energetic" cell death (as a direct result of ischemia) versus delayed degeneration of the axoglial unit (as part of a postischemic molecular cascade). Developmental studies of axoglial white matter signaling clearly indicate that a loss of 1 protein or 1 component in this cellular axis produces a delayed overall destruction in white matter [78-80]. Determination of the contributions of direct ischemic white matter cell death versus secondary white matter degeneration after ischemic loss of axons or glia will prove essential to understanding and treating white matter stroke.

Acknowledgments This work was supported by the National Institute of Neurological Disease and Stroke (grants NS053957 to STC and NS065723 to STC and JDH), the National Institute of Neurological Disease and Stroke National Research Service Award (F31NS076025 to EGS), and the American Heart Association Western States Affiliate Pre-doctoral fellowship to EGS. Support was provided to STC from Biogen Idec and Oncocyte Inc. for studies unrelated to those reported in this article.

Required Author Forms Disclosure forms provided by the authors are available with the online version of this article.

\section{References}

1. Bamford J, Sandercock P, Dennis M, Burn J, Warlow C. Classification and natural history of clinically identifiable subtypes of cerebral infarction. Lancet 1991;337:1521-1526.

2. Schneider AT, Kissela B, Woo D, et al. Ischemic stroke subtypes: a population-based study of incidence rates among blacks and whites. Stroke 2004;35:1552-1556.

3. Matsusue E, Sugihara S, Fujii S, et al. White matter changes in elderly people: MR-pathologic correlations. Magn Reson Med Sci 2006;5:99-104.

4. Gouw AA, van der Flier WM, Pantoni L, et al. LADIS Study Group. On the etiology of incident brain lacunes: longitudinal observations from the LADIS study. Stroke 2008;39:3083-3085.

5. Hainsworth AH, Markus HS. Do in vivo experimental models reflect human cerebral small vessel disease? A systematic review. J Cereb Blood Flow Metab 2008;28:1877-1891.

6. Román GC. On the history of lacunes, etat criblé, and the white matter lesions of vascular dementia. Cerebrovasc Dis 2002; (suppl 2)13:1-6.

7. Marie P. On lacunar foci of disintegration and other cavities of the brain. Rev Méd 1901;21:281-298. 
8. Román GC, Erkinjuntti T, Wallin A, et al. Subcortical ischaemic vascular dementia. Lancet Neurol 2002;1:426-436.

9. Vinters HV, Carmichael ST. The impact of cerebral small vessel disease on cognitive impairment and rehabilitation. In: Stuss DT, Winocur G, Robertson IH, eds. Cognitive neurorehabilitation: evidence and application. Cambridge Univ Press: Cambride 2008;360-375.

10. Srikanth V, Beare R, Blizzard L, et al. Cerebral white matter lesions, gait, and the risk of incident falls: a prospective population-based study. Stroke 2009;40:175-180.

11. Jokinen H, Gouw AA, Madureira S, et al. LADIS Study Group. Incident lacunes influence cognitive decline: the LADIS study. Neurology 2011;76:1872-1878.

12. Vernooij MW, Ikram MA, Tanghe HL, et al. Incidental findings on brain MRI in the general population. N Engl J Med 2007;357:18211828.

13. Knopman DS, Penman AD, Catellier DJ, et al. Vascular risk factors and longitudinal changes on brain MRI: the ARIC study. Neurology 2011;76:1879-1885.

14. Lammie GA. Hypertensive cerebral small vessel disease and stroke. Brain Pathol 2002;12:358-370.

15. Davis SM, Donnan GA. Why lacunar syndromes are different and important. Stroke 2004;35:1780-1781.

16. Waxman SG. Axonal dysfunction in chronic multiple sclerosis: meltdown in the membrane. Ann Neurol 2008;63:411-413.

17. Stys PK, Lesiuk H. Correlation between electrophysiological effects of mexiletine and ischemic protection in central nervous system white matter. Neuroscience 1996;71:27-36.

18. Fernando MS, Simpson JE, Matthews F, et al. MRC Cognitive Function and Aging Neuropathology Study Group. White matter lesions in an unselected cohort of the elderly: molecular pathology suggests origin from chronic hypoperfusion injury. Stroke 2006;37:1391-1398.

19. Jellinger KA, Attems J. Neuropathological evaluation of mixed dementia. J Neurol Sci 2007;257:80-87.

20. Wardlaw JM, Sandercock PA, Dennis MS, Starr J. Is breakdown of the blood-brain barrier responsible for lacunar stroke, leukoaraiosis, and dementia? Stroke 2003;34:806-812.

21. Verhaar MC, Strachan FE, Newby DE, et al. Endothelin-A receptor antagonist-mediated vasodilatation is attenuated by inhibition of nitric oxide synthesis and by endothelin-B receptor blockade. Circulation 1998;97:752-756.

22. Fuxe K, Cintra A, Andbjer B, Anggard E, Goldstein M, Agnati LF. Centrally administered endothelin-1 produces lesions in the brain of the male rat. Acta Physiol Scand 1989;37:155-156.

23. Hughes PM, Anthony DC, Ruddin M, et al. Focal lesions in the rat central nervous system induced by endothelin-1. J Neuropathol Exp Neurol 2003;62:1276-1286.

24. Sozmen EG, Kolekar A, Havton LA, Carmichael ST. A white matter stroke model in the mouse: axonal damage, progenitor responses and MRI correlates. J Neurosci Methods 2009;180:261-272.

25. Souza-Rodrigues RD, Costa AMR, Lima RR, Dos Santos CD, Picanço-Diniz CW, Gomes-Leal W. Inflammatory response and white matter damage after microinjections of endothelin-1 into the rat striatum. Brain Res 2008;1200:78-88.

26. Frost SB, Barbay S, Mumert ML, Stowe AM, Nudo RJ. An animal model of capsular infarct: endothelin-1 injections in the rat. Behav Brain Res 2006;169:206-211.

27. Newman TA, Woolley ST. T-cell- and macrophage-mediated axon damage in the absence of a CNS-specific immune response: involvement of metalloproteinases. Brain 2001;124:2203-2214.

28. Petzold A, Gveric D, Groves M, Schmierer K, Grant D, Chapman M. Phosphorylation and compactness of neurofilaments in multiple sclerosis: indicators of axonal pathology. Exp Neurol 2008;213:326335 .
29. Whitehead SN, Hachinski VC, Cechetto DF. Interaction between a rat model of cerebral ischemia and beta-amyloid toxicity: inflammatory responses. Stroke 2005;36:107-112.

30. Wang Y, Jin K, Greenberg DA. Neurogenesis associated with endothelin-induced cortical infarction in the mouse. Brain Res 2007;1167:118-122.

31. Horie N, Maag AL, Hamilton SA, Shichinohe H, Bliss TM, Steinberg GK. Mouse model of focal cerebral ischemia using endothelin-1. J Neurosci Methods 2008;173:286-290.

32. Wiley KE, Davenport AP. Endothelin receptor pharmacology and function in the mouse: comparison with rat and man. J Cardiovasc Pharmacol 2004; (suppl 1)44:S4-S6.

33. Koyama Y, Takemura M, Fujiki K, Ishikawa N, Shigenaga Y, Baba A. BQ788, an endothelin ET(B) receptor antagonist, attenuates stab wound injury-induced reactive astrocytes in rat brain. Glia 1999;26:268-271.

34. Gadea AS, Schinelli S, Gallo V. Endothelin-1 regulates astrocyte proliferation and reactive gliosis via a $\mathrm{JNK} / \mathrm{c}-\mathrm{Jun}$ signaling pathway. J Neurosci 2008;28:2394-2408.

35. Sanchez-Alvarez R, Tabernero A, Medina JM. Endothelin-1 stimulates the translocation and upregulation of both glucose transporter and hexokinase in astrocytes: relationship with gap junctional communication. J. Neurochem 2004;89:703-714.

36. Gadea A, Aguirre A, Haydar TF, Gallo V. Endothelin-1 regulates oligodendrocyte development. J Neurosci 2009;29:10047-10062.

37. He Z, Yamawaki T, Yang S, Day AL, Simpkins JW, Naritomi H. Experimental model of small deep infarcts involving the hypothalamus in rats: changes in body temperature and postural reflex. Stroke 1999;30:2743-2751.

38. He Z, Yang SH, Naritomi H, Yamawaki T, et al. Definition of the anterior choroidal artery territory in rats using intraluminal occluding technique. J Neurol Sci 2000;182:16-28.

39. Tanaka Y, Imai H, Konno K, et al. Experimental model of lacunar infarction in the gyrencephalic brain of the miniature pig: neurological assessment and histological, immunohistochemical, and physiological evaluation of dynamic corticospinal tract deformation. Stroke 2006;39:205-212.

40. Ni J, Ohta H, Matsumoto K, Watanabe H. Progressive cognitive impairment following chronic cerebral hypoperfusion induced by permanent occlusion of bilateral carotid arteries in rats. Brain Res 1994;653:231-236.

41. Farkas E, Institoris A, Domoki F, Mihaly A, Luiten PG, Bari F. Diazoxide and dimethyl sulphoxide prevent cerebral hypoperfusionrelated learning dysfunction and brain damage after carotid artery occlusion. Brain Res 2004;1008:252-260.

42. Wakita H, Tomimoto H, Akiguchi I, Kimura J. Glial activation and white matter changes in the rat brain induced by chronic cerebral hypoperfusion: an immunohistochemical study. Acta Neuropathol 1994;87:484-492.

43. Hattori H, Takeda M, Kudo T, Nishimura T, Hashimoto S. Cumulative white matter changes in the gerbil brain under chronic cerebral hypoperfusion. Acta Neuropathol 1992;84:437-442.

44. Kudo T, Takeda M, Tanimukai S, Nishimura T. Neuropathologic changes in the gerbil brain after chronic hypoperfusion. Stroke 1993;24:259-265.

45. Kurumatani T, Kudo T, Ikura Y, Takeda M. White matter changes in the gerbil brain under chronic cerebral hypoperfusion. Stroke 1998;29:1058-1062.

46. Shibata M, Ohtani R, Ihara M, Tomimoto H. White matter lesions and glial activation in a novel mouse model of chronic cerebral hypoperfusion. Stroke 2004;35:2598-2603.

47. Shibata M, Yamasaki N, Miyakawa T, et al. Selective impairment of working memory in a mouse model of chronic cerebral hypoperfusion. Stroke 2007;38:2826-2832.

48. Hollander W, Prusty S, Kirkpatrick B, Paddock J, Nagraj S. Role of hypertension in ischemic heart disease and cerebral vascular 
disease in the cynomolgus monkey with coarctation of the aorta. Circ Res 1977;40:170-183.

49. Kemper T, Moss MB, Hollander W, Prusty S. Microinfarction as a result of hypertension in a primate model of cerebrovascular disease. Acta Neuropathol 1999;98:295-303.

50. Kemper TL, Blatt GJ, Killiany RJ, Moss MB. Neuropathology of progressive cognitive decline in chronically hypertensive rhesus monkeys. Acta Neuropathol 2001;101:145-153.

51. Kantachuvesiri S, Fleming S, Peters J, et al. Controlled hypertension, a transgenic toggle switch reveals differential mechanisms underlying vascular disease. J Biol Chem 2001;276:36727-36733.

52. Collidge TA, Lammie GA, Fleming S, Mullins JJ. The role of the renin-angiotensin system in malignant vascular injury affecting the systemic and cerebral circulations. Prog Biophys Mol Biol 2004;84:301-319.

53. Baumbach GL, Sigmund CD, Faraci FM. Cerebral arteriolar structure in mice overexpressing human renin and angiotensinogen. Hypertension 2003;41:50-55.

54. Iida S, Baumbach GL, Lavoie JL, Faraci FM, Sigmund CD, Heistad DD. Spontaneous stroke in a genetic model of hypertension in mice. Stroke 2005;36:1253-1258.

55. Okamoto K, Hazama F, Yamori Y, Haebara H, Nagaoka A. Pathogenesis and prevention of stroke in spontaneously hypertensive rats. Clin Sci Mol Med 1975;(suppl 2)161s-163s.

56. Yamori Y, Horie R, Handa H, Sato M, Fukase M. Pathogenetic similarity of strokes in stroke-prone spontaneously hypertensive rats and humans. Stroke 1976;7:46-53.

57. Yamori Y, Horie R. Developmental course of hypertension and regional cerebral blood flow in stroke-prone spontaneously hypertensive rats. Stroke 1977;8:456-461.

58. Tagami M, Nara Y, Kubota A, et al. Ultrastructural characteristics of occluded perforating arteries in stroke-prone spontaneously hypertensive rats. Stroke 1987;18:733-740.

59. Ogata J, Fujishima M, Tamaki K, Nakatomi Y, Ishitsuka T, Omae $\mathrm{T}$, Vascular changes underlying cerebral lesions in stroke-prone spontaneously hypertensive rats. A serial section study. Acta Neuropathol 1981;54:183-188.

60. Fredriksson K, Auer RN, Kalimo H, Nordborg C, Olsson Y, Johansson BB. Cerebrovascular lesions in stroke-prone spontaneously hypertensive rats. Acta Neuropathol 1985;68:284-294.

61. Fredriksson K, Nordborg C, Kalimo H, Olsson Y, Johansson BB. Cerebral microangiopathy in stroke-prone spontaneously hypertensive rats. An immunohistochemical and ultrastructural study. Acta Neuropathol 1988;75:241-252.

62. Ruchoux MM, Domenga V, Brulin P, et al. Transgenic mice expressing mutant Notch3 develop vascular alterations characteristic of cerebral autosomal dominant arteriopathy with subcortical infarcts and leukoencephalopathy. Am J Pathol 2003;162:329-342.

63. Gould DB, Phalan FC, Breedveld GJ, et al. Mutations in Col4a1 cause perinatal cerebral hemorrhage and porencephaly. Science 2005;308:1167-1171.
64. Weiller C, Ramsay SC, Wise RJ, Friston KJ, Frackowiak RS. Individual patterns of functional reorganization in the human cerebral cortex after capsular infarction. Ann Neurol 1993;33:181-189.

65. Zhu X, Bergles DE, Nishiyama A. NG2 cells generate both oligodendrocytes and gray matter astrocytes. Development 2007;135:145157.

66. Franklin RJ, Ffrench-Constant C. Remyelination in the CNS: from biology to therapy. Nat Rev Neurosci 2008;9:839-855.

67. Fancy SP, Zhao C, Franklin RJ. Increased expression of Nkx2.2 and Olig2 identifies reactive oligodendrocyte progenitor cells responding to demyelination in the adult CNS. Mol Cell Neurosci 2004;27:247-254.

68. Arnett HA, Wang Y, Matsushima GK, Suzuki K, Ting JP. Functional genomic analysis of remyelination reveals importance of inflammation in oligodendrocyte regeneration. J Neurosci 2003;23:9824-9832.

69. Penderis J, Woodruff RH, Lakatos A, et al. Increasing local levels of neuregulin (glial growth factor-2) by direct infusion into areas of demyelination does not alter remyelination in the rat CNS. Eur J Neurosci 2003;18:2253-2264.

70. Sim FJ, Lang JK, Waldau B, Roy NS, Schwartz TE, Pilcher WH. Complementary patterns of gene expression by human oligodendrocyte progenitors and their environment predicts determinants of progenitor maintenance and differentiation. Ann Neurol 2006;59;763779 .

71. Wilczak N, Chesik D, Hoekstra D, De Keyser J. IGF binding protein alterations on periplaque oligodendrocytes in multiple sclerosis: implications for remyelination. Neurochem Int 2008;52:1431-1435.

72. McIver SR, Muccigrosso M, Gonzales ER, et al. Oligodendrocyte degeneration and recovery after focal cerebral ischemia. Neuroscience 2010;169:1364-1375.

73. Stirling DP, Stys PK. Mechanisms of axonal injury: internodal nanocomplexes and calcium deregulation. Trends Mol Med 2010;16:160-170.

74. Dewar D, Underhill SM, Goldberg MP. Oligodendrocytes and ischemic brain injury. J Cereb Blood Flow Metab 2003;23:263274.

75. McTigue DM, Tripathi RB. The life, death, and replacement of oligodendrocytes in the adult CNS. J Neurochem 2008;107:1-19.

76. Pantoni L. Experimental approaches to white matter disease. Dement Geriatr Cogn Disord 1998;(suppl 1):20-24.

77. Pantoni L, Garcia JH, Gutierrez JA. Cerebral white matter is highly vulnerable to ischemia. Stroke 1996;27:1641-1647.

78. Bhat MA, Rios JC, Lu Y, et al. Axon-glia interactions and the domain organization of myelinated axons requires neurexin IV/ Caspr/Paranodin. Neuron 2001;30:369-383.

79. Boyle ME, Berglund EO, Murai KK, Weber L, Peles E, Ranscht B. Contactin orchestrates assembly of the septate-like junctions at the paranode in myelinated peripheral nerve. Neuron 2001;30:385-397.

80. Sherman DL, Brophy PJ. Mechanisms of axon ensheathment and myelin growth. Nat Rev Neurosci 2005;6:683-690. 